\title{
Recombinant fusion proteins FPTD-Grb2-SH2 and FPTD-Grb2-SH2M inhibit the proliferation of breast cancer cells in vitro
}

\author{
JIKAI YIN $^{1 *}$, LIJUAN YUAN $^{1 *}$, ZIYU LIU $^{2 *}$, FANGLIN ZHANG $^{2}$, XIANLI HE ${ }^{1}$, ZHIKAI XU $^{2}$, \\ QING WANG ${ }^{1}, X^{2} I N D^{1}, X^{\prime} G^{\prime} A N W^{2}$ and JIANGUO LU ${ }^{1}$ \\ ${ }^{1}$ Department of General Surgery, Tangdu Hospital of The Fourth Military Medical University, Ba'qiao, Xi'an, Shaanxi 710038; \\ ${ }^{2}$ Department of Microbiology, The Fourth Military Medical University, Chang'le, Xi'an, Shaanxi 710032, P.R. China
}

Received February 12, 2014; Accepted March 21, 2014

DOI: $10.3892 /$ or.2014.3130

\begin{abstract}
Growth factor receptor bound protein 2 (Grb2) is a key adaptor performing a principal role in the oncogenic Ras signaling pathway. In the present study, we generated two fusion proteins. One contained an Src homology 2 (SH2) domain of Grb2, a signal peptide sequence, FLAG-tag sequence, PTD region and we named it FPTD-Grb2-SH2, while the other contained one mutant $\mathrm{SH} 2$ domain, added to a signal peptide sequence, FLAG-tag sequence, PTD region and we named it FPTD-Grb2-SH2M. Western blot analysis and immunofluorescence assay were used to investigate the expression and location of the fusion proteins in breast cancer cells. The proliferation and migration of the cells were estimated by MTT and Transwell cell migration assays, respectively. Flow cytometric analysis was performed to evaluate the apoptosis of the breast cancer cells. The recombinant proteins FPTD-Grb2-SH2 and FPTD-Grb2-SH2M were successfully expressed in the breast cancer cell lines regardless of HER2-phenotype, and they suppressed breast cancer cell growth and migration as expected from the lack of SH3 domain. Both FPTD-Grb2-SH2 and FPTD-Grb2-SH2M exhibited significant toxicity to breast cancer cells. The present study demonstrated that the recombinant proteins FPTD-Grb2-SH2 and FPTD-Grb2-SH2M may be used for anticancer drug development.
\end{abstract}

Correspondence to: Dr Jianguo Lu, Department of General Surgery, Tangdu Hospital of The Fourth Military Medical University, Xin'si Road, Ba'qiao, Xi'an, Shaanxi 710038, P.R. China E-mail: lujguo@hotmail.com

Professor Xing'an Wu, Department of Microbiology, The Fourth Military Medical University, Chang'le, Xi'an, Shaanxi 710032, P.R. China

E-mail: wuxingan@fmmu.edu.cn

*Contributed equally

Key words: breast cancer, growth factor receptor bound protein 2, Src-homology 2 domain, MCF-7, MDA-MB-231

\section{Introduction}

Breast cancer is a major health issue worldwide. It was estimated that approximately 234,580 individuals were diagnosed with breast cancer, and more than 40,030 died from this disease in the US in 2013 (1). In China, breast cancer is currently the most common malignancy and the sixth leading cause of cancer-related mortality in women (2). Epidermal growth factor receptor (EGFR) and ErbB2 (HER2) are both receptor tyrosine kinases (RTKs), which play important roles in the regulation of cell proliferation, motility and apoptosis. Accumulating evidence has confirmed that they are major and attractive targets for cancer therapy. Previous studies have reported that EGFR and ErbB2 are overexpressed in $~ 40-50 \%$ of breast cancer cases (3), and increased expression of EGFR or ErbB2 is associated with breast cancer growth and the poor survival and prognosis of breast cancer patients (3). To date, a number of agents have been developed to target EGFR or ErbB2 that result in tumor cell growth arrest and apoptosis (4-6), most of which have focused on the inhibition of ligand binding or tyrosine phosphorylation and have achieved success. However, EGFR and ErbB2 mutations in cancers make the development of novel agents targeting the EGFR or ErbB2 signaling pathway urgent for cancer therapy (7-9).

Growth factor receptor bound protein 2 (Grb2) is a key adaptor binding to EGFR and ErbB2, which in turn binds to SOS and activates transduction of cellular responses downstream of Ras RTKs through the Ras-ERK signaling cascade (10). It consists of a central Src homology 2 (SH2) domain and two Src homology 3 (SH3) domains. The N-terminal SH3 domain recognizes proline-rich sequences adopting a type II polyproline (PPII) helix, while the C-terminal SH3 domain recognizes sequences conforming $\mathrm{P}-\mathrm{X}-\mathrm{I} / \mathrm{L} / \mathrm{V} / \mathrm{-D} / \mathrm{N}-\mathrm{R}-\mathrm{X}-\mathrm{X}-\mathrm{K}-\mathrm{P}$ motif (11). The SH2 domains recognize tyrosine phosphorylated sequences expediting phosphorylation-dependent interactions of proteins resulting in signal propagation $(12,13)$. Obvious overexpression of Grb2 was observed in breast cancer cell lines and tissue samples $(14,15)$. Grb2 links RTKs to the Ras signaling pathway, which controls major signaling routes of cell proliferation, differentiation and survival. It has been reported that downregulation of Grb2 suppresses 
the differentiation of mouse embryonic stem cells (16) and silencing Grb2 expression reduces cell growth in vitro (17). It has been suggested that Grb2 is a potential target for the development of therapeutic agents.

$\mathrm{SH} 2$ domains are found in many intercellular signal-transduction proteins. SH2 is involved in the development of a wide range of human diseases including cancers, diabetes and immunodeficiencies (18). SH2 is a relatively small protein module of $\sim 100$ amino acids, which facilitates activation of intracellular signaling by interacting with phosphorylated tyrosine containing peptide sequences $(19,20)$. The binding selectivity of $\mathrm{SH} 2$ domains is critical for the fidelity and specificity of cellular signal transduction pathways (21-24). Considerable effort has been devoted to study the pathogenic function of SH2 domains. The fusion Grb2-SH2 protein was found to inhibit EGFR-Grb2 signaling in squamous cells and may have the potential to treat EGFR-activated cancer (7). The prokaryotic recombined fusion Grb2-SH2 protein was confirmed to inhibit the proliferation of breast cancer cells in our previous study (25).

To investigate the function of the Grb2-SH2 domain, we generated two fusion proteins. One contained one $\mathrm{SH} 2$ domain of Grb2, together with a signal peptide sequence, the PTD region, and FLAG-tag sequence. The other one consisted of a mutant $\mathrm{SH} 2$ domain, signal peptide sequence, PTD region and a FLAG-tag sequence. The PTD sequence was reported in a previous study (25). In the present study, we constructed eukaryotic expression vectors containing the recombinant gene sequences and they were expressed in breast cancer cell lines, for which the biological behavior was also investigated.

\section{Materials and methods}

Cell culture. E. coli JM109 (Invitrogen, Carlsbad, CA, USA) were stored at $-70^{\circ} \mathrm{C}$ and cultured in Luria-Bertani broth medium (LB). The pUC-57 and pIRES2-EGFP were commercial products (BD Biosciences, Clontech, CA, USA) and stored at $-20^{\circ} \mathrm{C}$. The breast cancer cell lines HER2-positive MCF-7 and HER2-negative MDA-MB-231 were preserved in the Department of Molecular Biology of the Fourth Military Medical University. The cells were cultured in Dulbecco's modified Eagle's medium (DMEM; Sigma-Aldrich, St. Louis, MO, USA) supplemented with $10 \%$ fetal bovine serum (FBS), 50 units $/ \mathrm{ml}$ penicillin and $50 \mu \mathrm{g} / \mathrm{ml}$ streptomycin (FBS; Gibco Invitrogen Corp., Carlsbad, CA, USA). Cell cultures were maintained in a humidified $5 \% \mathrm{CO}_{2}$ atmosphere at $37^{\circ} \mathrm{C}$.

Expression vector construction. The fusion FPTD-Grb2-SH2 DNA sequence was designed based on the native Grb2-SH2 coding DNA sequence as previously described (25). It contained one signal peptide DNA sequence (5'-ATGAAGCA CCTTTGGTTCTTCCTTCTTCTTGTAGCAGCACCAAGG TGGGTACTTTCG-3'); one FLAG-tag DNA sequence (5'-GA CTACAAGGACGATGATGACAAG-3'); one PTD-transduction domain DNA sequence (5'-TATGGTAGGAAG AAACGTCGACAGCGTCGTCGG-3') and one Grb2-SH2 domain DNA sequence, which was synthesized by Sangon Biotech Co. The artificial DNA fragment was digested with $B a m \mathrm{HI}$ and EcoRI into the pUC-57 plasmid and subsequently subcloned into digested pIRES2-EGFP to construct the expression vector, which was named pIRES-FPTD-Grb2SH2. Meanwhile, a mutant DNA fragment was designed as a contrast. It was amplified by polymerase chain reaction using a forward primer (5'-TGGTTTTTTGGCAAAATCCCCT CTTTGAATGAGC-3') and a reverse primer (5'-ATCCACC AGCTCATTCAAAGAGGGGATTTTGCC-3'). This DNA fragment was subsequently inserted into digested pUC-57 and pIRES2-EGFP. The recombinant expression vector was named pIRES-FPTD-Grb2-SH2M. All insertions were confirmed by DNA sequencing and BLAST program (http://www.ncbi.nlm. nih.gov/BLAST/). The recombinant plasmids were kept in JM109 and stored at $-70^{\circ} \mathrm{C}$.

Transfection. Cells were transfected with the recombinant plasmidspIRES-FPTD-Grb2-SH2 andpIRES-FPTD-Grb2-SH2M. The breast cancer cells were seeded at $2 \times 10^{5} /$ well in 6 -well plates for $\sim 24 \mathrm{~h}$ until $80 \%$ confluency was achieved. Cell transfections were performed using X-tremeGENE HP DNA transfection reagent (Roche, Germany) according to the manufacturer's instructions. Plasmid DNA ( $2 \mu \mathrm{g})$ was mixed with the transfection reagent, and then the complex was added to the cell cultured in fresh medium. Transfected cells were incubated for 36 and $48 \mathrm{~h}$. The untransfected cells cultured in the same condition were set as controls. The cells incubated with the transfection reagent and plasmid pIRES2-EGFP were also included in the experiments. At the end of each transfection period, cells were collected for the following measurement [immunofluorescence assay, western blot analysis, 3-[4,5-dimethylthiazol-2-yl]2,5-diphenyltetrazolium bromide (MTT) assay, cell migration assay and flow cytometric detection].

Western blot analysis. Cell lysates containing $30 \mu \mathrm{g}$ protein were subjected to SDS-PAGE on a $15 \%$ gel and transferred onto a PVDF membrane (Bio-Rad). They were probed by a primary antibody against FLAG-tag (Qiagen; 1:2,000) with $2.5 \%$ milk in TBS. HRP-labeled secondary antibodies were detected using ECL detection kit (GE Healthcare), and the target bands were visualized using the ChemiDoc ${ }^{\mathrm{TM}}$ MP detection system (Bio-Rad Laboratories, Inc.).

Immunofluorescence assay. Cells on coverslips were fixed in 4\% paraformaldehyde and permeabilized in PBS with $0.1 \%$ Triton X-100. After blocking the non-specific binding in $2 \%$ normal sheep serum in PBS, the coverslips were incubated with a rabbit anti-FLAG-tag polyclonal antibody (Santa Cruz Biotechnology, Santa Cruz, CA, USA) at a 1:400 dilution overnight at $4^{\circ} \mathrm{C}$. The cells were then washed with PBS and incubated with a fluorescence-labeled secondary antibody (Dako, Glostrup, Denmark) for $3 \mathrm{~h}$. The specific immunoreactivity was observed under a reflected light fluorescence microscope (BH2-RFC; Olympus, Tokyo, Japan).

Cell proliferation assay. The MTT assay was used to evaluate cell proliferation following transfection. Cells were harvested and plated in 96-well cell plates $\left(2 \times 10^{4}\right.$ cells/well) in sextuplicate. Aliquots $(20 \mu \mathrm{l})$ of $5 \mathrm{mg} / \mathrm{ml}$ MTT (Sigma) in PBS were added to each well and incubated for $4 \mathrm{~h}$ at $37^{\circ} \mathrm{C}$; $150 \mu 1$ of $\mathrm{Me}_{2} \mathrm{SO}$ was subsequently added. The absorbance (A) values were measured on a Sunrise microplate reader (Tecan, Groedig, Austria) at $490 \mathrm{~nm}$. 


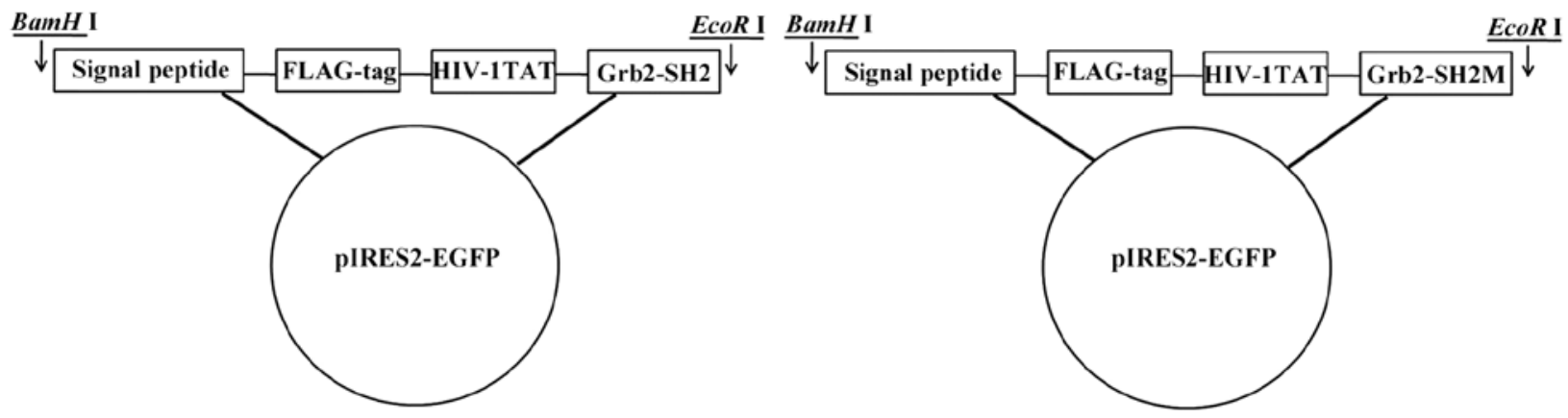

Figure 1. Construction of pIRES-FPTD-Grb2-SH2 and pIRES-FPTD-Grb2-SH2M. The FPTD-Grb2-SH2 coding frame is presented with a signal peptide sequence, FLAG-tag and HIV-1 TAT domain. Grb2, growth factor receptor bound protein 2; SH2, Src homology 2.

Cell migration assay. The cells $\left(2 \times 10^{5}\right)$ in $200 \mu 1$ medium free of serum were placed in the upper chamber of Transwell cell culture inserts ( $8-\mu \mathrm{m}$ pore size; Corning Life Sciences). The lower chamber contained $500 \mu \mathrm{l}$ complete medium (DMEM with $10 \% \mathrm{FBS})$. Migration was carried out for $24 \mathrm{~h}$ at $37^{\circ} \mathrm{C}$ in a $5 \% \mathrm{CO}_{2}$ atmosphere. Cells that had migrated into the lower chamber were quantified by counting in a Neubauer counting chamber using an inverted cell culture microscope (Olympus). The migration inhibition rate was calculated using the formula: Migration inhibition rate $=[(1-$ transfected cells/untransfected cells) x $100 \%]$.

Analysis of cell apoptosis. Cells were stained with Annexin V-FITC and PI, and flow cytometric analysis was performed using the Elite ESP flow cytometer (FACSCalibur; Becton-Dickinson Immunocytometry Systems, San Jose, CA, USA) for apoptosis analysis according to the manufacturer's guidelines. The data were analyzed using the CellQuest Pro Software (BD Biosciences, San Jose, CA, USA).

Statistical analysis. Statistical analysis was performed using the SPSS 19.0 software package for Windows. P-values $<0.05$ were considered to indicate statistically significant results.

\section{Results}

Sequence synthesis, expression and identification. The successful construction of the expression vectors pIRESFPTD-Grb2-SH2 and pIRES-FPTD-Grb2-SH2M was confirmed by restriction mapping (Fig. 1). The result of the automatic sequencing was consistent with the expected FPTD-Grb2-SH2 DNA fragment (388 bp) and its mutant (208 bp). The expression vectors were transfected into breast cancer cells, respectively, with untransfected cells, transfection reagent and empty vector used as controls. The average transfection efficiency was $\sim 70 \%$ as estimated by fluorescencepositive cells under a florescence microscope after $36 \mathrm{~h}$. The transfected cells were incubated for 36 and $48 \mathrm{~h}$, respectively. Immunofluorescence assay with the anti-FLAG-tag antibody revealed that FPTD-Grb2-SH2 and FPTD-Grb2-SH2M proteins were expressed in the MCF-7 and MDA-MB-231 cells. As shown in Fig. 2, both the recombinants were mainly located in the cytoplasm, indicating that signal peptide and HIV-1 TAT48-60 helped the target peptides to pass through the nuclear membranes in living cells.
Protein expression levels were further determined by western blotting (Fig. 3). A statistically significant stronger expression of FPTD-Grb2-SH2 compared to FPTD-Grb2-SH2M was observed in both breast cancer cell lines $(\mathrm{P}<0.05)$. Expression of FPTD-Grb2-SH2 protein increased when transfected MDA-MB-231 cells were incubated for $48 \mathrm{~h}$, while it decreased in MCF-7 cells as shown in Fig. 3E.

Growth inhibition of fusion FPTD-Grb2-SH2 and fusion FPTD-Grb2-SH2M proteins in the breast cancer MCF-7 and $M D A-M B-231$ cell lines. The recombinant plasmids pIRES-FPTD-Grb2-SH2 and pIRES-FPTD-Grb2-SH2M were transfected into breast cancer cell lines MCF-7 and MDA-MB-231 to investigate whether the new genes could affect the proliferation of breast cancer cells in vivo. The growth inhibitory effects of FPTD-Grb2-SH2 and its mutant were observed in both breast cancer cells (Fig. 4). FPTD-Grb2-SH2 exhibited a significantly stronger inhibitory effect on the breast cancer cells. After incubation for $48 \mathrm{~h}$, a decreased rate of cell proliferation inhibition was detected in the transfected cells, when compared to the transfected cells incubated for $36 \mathrm{~h}(\mathrm{P}<0.05)$.

Inhibition of migratory ability by fusion FPTD-Grb2-SH2 and fusion FPTD-Grb2-SH2M proteins in the MCF-7 and $M D A-M B-231$ cells. As shown in Fig. 5, the migratory ability of the MCF-7 and MDA-MB-231 cells was reduced by recombinant FPTD-Grb2-SH2 and FPTD-Grb2-SH2M when compared to the empty vector control. Fig. 5A and B shows that the percentage of viable cells transfected with the fusion proteins was reduced by at least $\sim 15 \%$ as compared with the cells transfected with the empty vector. The migration inhibition rate was enhanced when the transfected cells were incubated for $48 \mathrm{~h}$. No significant difference was noted in the cells transfected with the empty vector control.

Recombinant FPTD-Grb2-SH2 and FPTD-Grb2-SH2M proteins induce the apotosis of $M C F-7$ and $M D A-M B-231$ cells. MCF-7 and MDA-MB-231 cells were stained with Annexin V-FITC and PI, and then subjected to flow cytometry. Fig. 6 reveals that both FPTD-Grb2-SH2 and its mutant induced apoptosis in the MCF-7 and MDA-MB-231 cells, when compared to the empty vector controls. However, marked induction of apoptosis of the MCF-7 cells was induced 

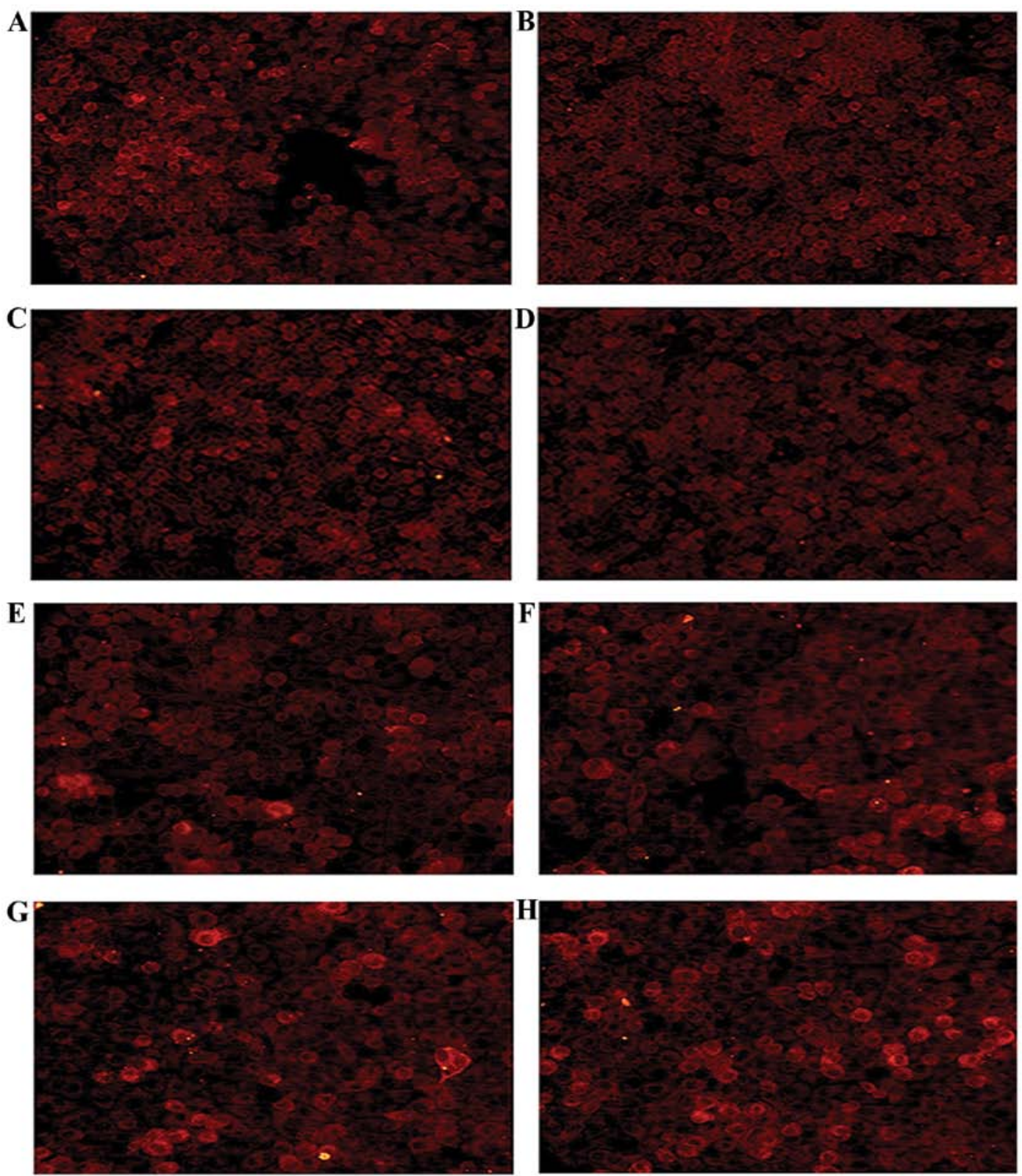

Figure 2. Immunofluorescence assay confirms the expression of FPTD-Grb2-SH2 and FPTD-Grb2-SH2M in breast cancer cell lines MDA-MB-231 and MCF-7. Images were captured under a x20 objective. (A) MDA-MB-231 cells with FPTD-Grb2-SH2 incubated for 36 h. (B) MDA-MB-231 cells with FPTD-Grb2-SH2 incubated for $48 \mathrm{~h}$. (C) MDA-MB-231 cells with FPTD-Grb2-SH2M incubated for $36 \mathrm{~h}$. (D) MDA-MB-231 cells with FPTD-Grb2-SH2M incubated for 48 h. (E) MCF-7 cells with FPTD-Grb2-SH2 incubated for 36 h. (F) MCF-7 cells with FPTD-Grb2-SH2 incubated for 48 h. (G) MCF-7 cells with FPTD-Grb2-SH2M incubated for $36 \mathrm{~h}$. (H) MCF-7 cells with FPTD-Grb2-SH2M incubated for $48 \mathrm{~h}$. Grb2, growth factor receptor bound protein 2; SH2, Src homology 2 .
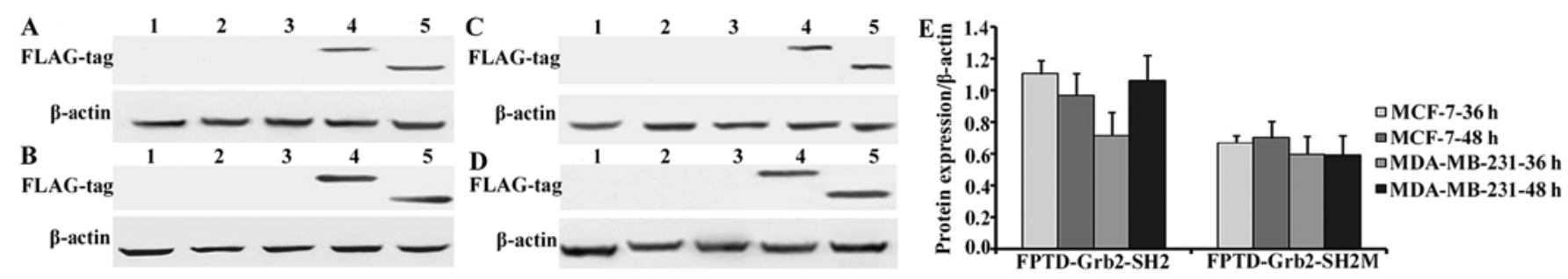

Figure 3. Identification of recombinant FPTD-Grb2-SH2 and FPTD-Grb2-SH2M in breast cancer cell lines MCF-7 and MDA-MB-231 by western blotting. $\beta$-actin expression was used as a loading control. (A) Transfected MCF-7 cells incubated for $36 \mathrm{~h}$. (B) Transfected MCF-7 cells incubated for $48 \mathrm{~h}$. (C) Transfected MDA-MB-231 cells incubated for 36 h. (D) Transfected MDA-MB-231 cells incubated for 48 h. Lane 1, cells without treatment; lane 2, cells with transfection reagent; lane 3, cells with empty vector; lane 4, cells with pIRES-FPTD-Grb2-SH2; lane 5, cells with pIRES-FPTD-Grb2-SH2M. (E) Western blot analysis of the recombinant FPTD-Grb2-SH2 and FPTD-Grb2-SH2M proteins. Data were normalized to $\beta$-actin. (Data are expressed as the mean value \pm standard deviation; $\mathrm{P}<0.05$ ). Grb2, growth factor receptor bound protein 2; SH2, Src homology 2 . 

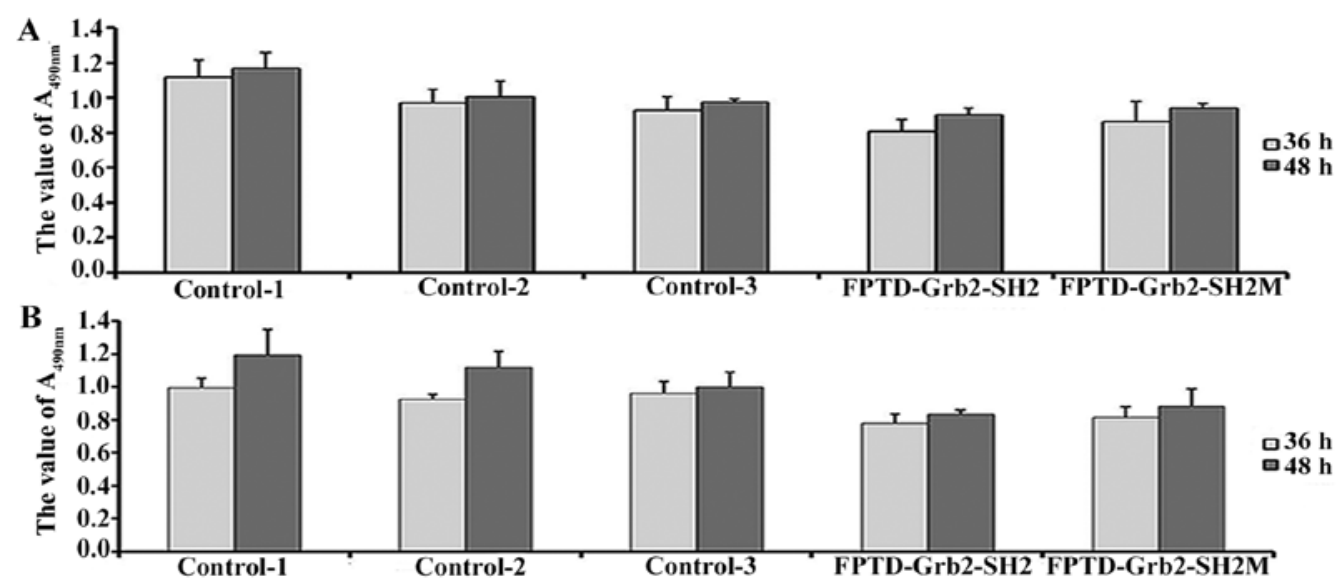

Figure 4. MTT assay showing the inhibition of the proliferation by FPTD-Grb2-SH2 and FPTD-Grb2-SH2M. Proliferation of (A) MCF-7 and (B) MDAMB-231 cells. Control-1, cells without treatment; control-2, cells with transfection reagent; control-3, cells with empty vector; FPTD-Grb2-SH2, cells with pIRES-FPTD-Grb2-SH2; FPTD-Grb2-SH2M, cells with pIRES-FPTD-Grb2-SH2M. Grb2, growth factor receptor bound protein 2; SH2, Src homology 2.
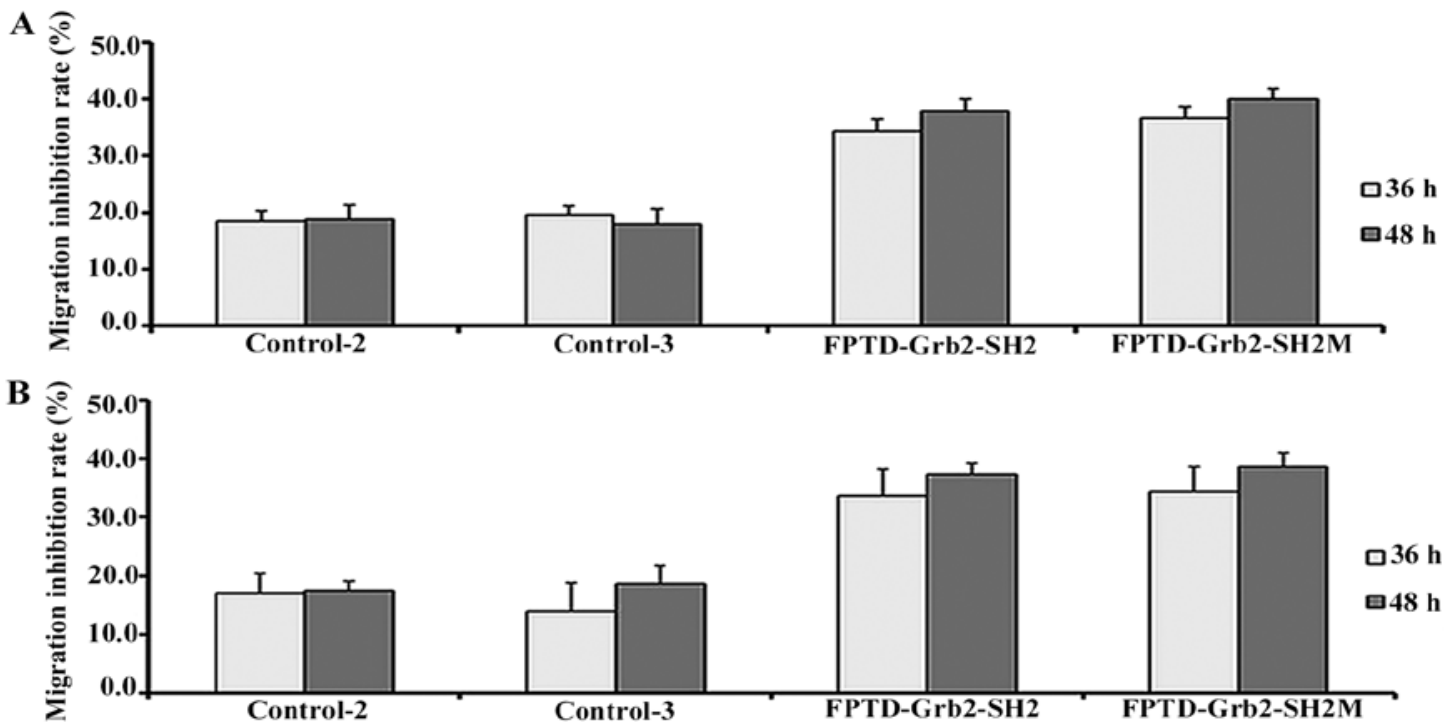

Figure 5. Migration inhibition by FPTD-Grb2-SH2 and FPTD-Grb2-SH2M proteins in MCF-7 and MDA-MB-231 cells. Cells without treatment were set as the untransfected cell control. Migration of (A) MCF-7 and (B) MDA-MB-231 cells. Control-2, cells with transfection reagent; control-3, cells with empty vector; FPTD-Grb2-SH2, cells with pIRES-FPTD-Grb2-SH2; FPTD-Grb2-SH2M, cells with pIRES-FPTD-Grb2-SH2M. Grb2, growth factor receptor bound protein 2; $\mathrm{SH} 2, \mathrm{Src}$ homology 2.

by FPTD-Grb2-SH2. Apoptosis proceeded in $59.62 \%$ of the infected MCF-7 cells at $36 \mathrm{~h}$ and $61.52 \%$ of infected MCF-7 cells at $48 \mathrm{~h}$, which was much higher than the percentage in the MDA-MB-231 cells (12.06 and 13.68\%, respectively) (Fig. 6). In addition, the ability to induce apoptosis exerted by FPTDGrb2-SH2 in both breast cell lines was much more obvious than that by FPTD-Grb2-SH2M.

\section{Discussion}

Grb2 is a dynamic downstream intermediary in the HER2 signaling pathways, which has been implicated directly in the pathogenesis of several specific human malignancies. In addition, overexpression of Grb2 is found in breast and bladder cancer cells $(26,27)$, which suggests that Grb2 can be a valid therapeutic target for cancer treatment. The Grb2-SH2 domain is a relatively conserved 100 amino acid modular unit, and disruption of Grb2-SH2 domain interactions can significantly inhibit the Grb2-mediated signaling pathway (28). Therefore, the Grb2-SH2 domain has been recognized as an ideal pharmaceutical target.

In our previous study, we expressed and purified a novel prokaryotic fusion $\mathrm{SH} 2$ protein and its mutant, which were successfully transduced into living breast cancer cells resulting in the inhibition of growth of these cells (25). In the present study, the eukaryotic fusion proteins containing the $\mathrm{SH} 2$ domain and the mutant with a signal peptide sequence, PTD domain sequence and FLAG-tag sequence were directly expressed in breast cancer cells, respectively. (The proteins were named FPTD-Grb2-SH2 and FPTD-Grb2-SH2M, respectively). The fusion proteins were small proteins with a molecular weight $<20 \mathrm{kDa}$. Automatic sequencing examined the target sequences to prevent mis-construction and expression. Unlike the prokaryotic mutant protein, FPTD- 


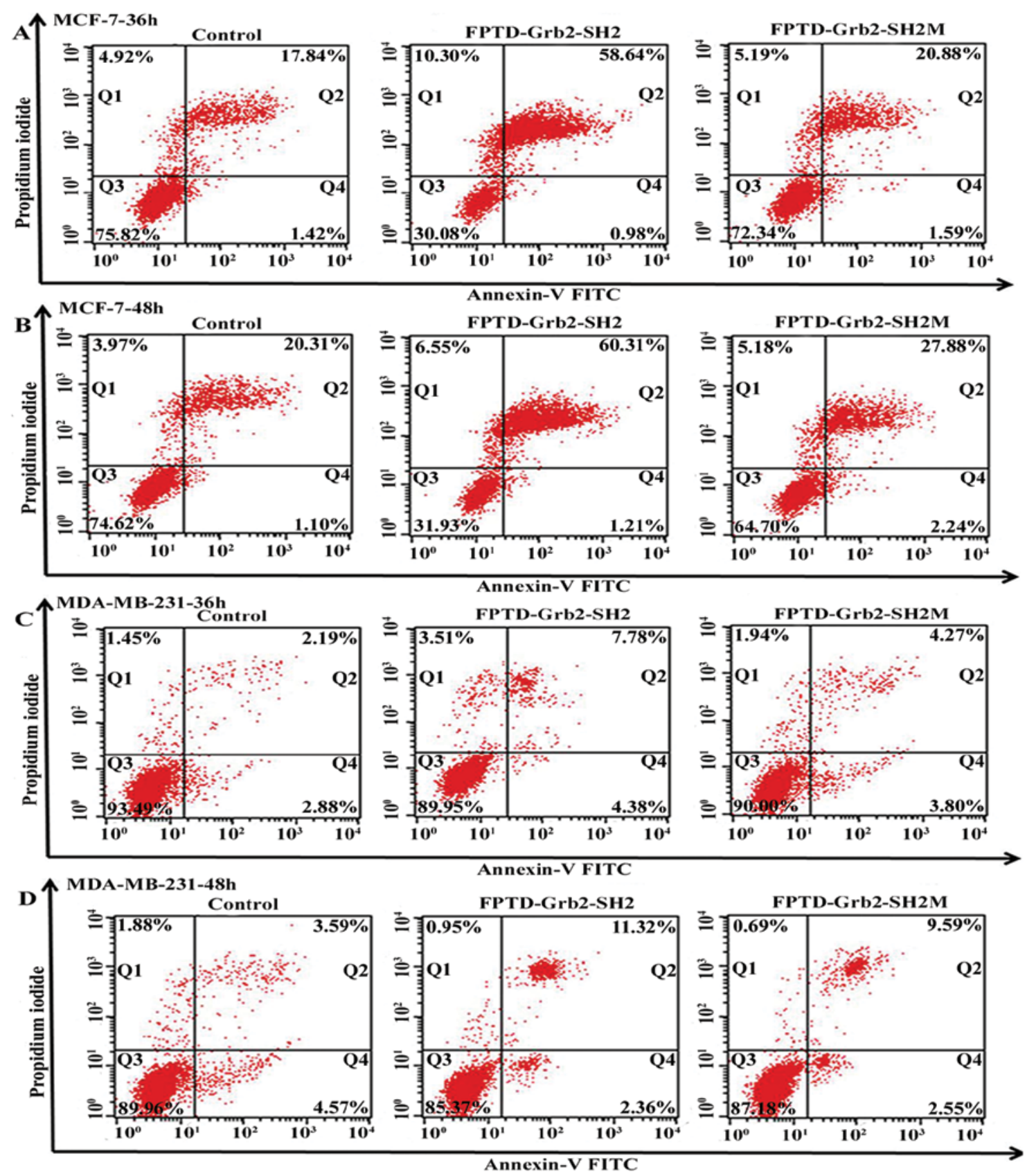

Figure 6. Apoptosis of breast cancer cells was confirmed by Annexin V-FITC, propidium iodide flow cytometry. Cells with the empty vector were set as control. (A) Transfected MCF-7 cells incubated for $36 \mathrm{~h}$. (B) Transfected MCF-7 cells incubated for $48 \mathrm{~h}$. (C) Transfected MDA-MB-231 cells incubated for $36 \mathrm{~h}$. (D) Transfected MDA-MB-231 cells incubated for $48 \mathrm{~h}$. Q1, necrotic cells; Q2, cells at late stage of apotosis; Q3, living cells; Q4, cells at early stage of apoptosis.

Grb2-SH2M also exerted growth inhibition in the breast cancer cell lines MCF-7 and MDA-MB-231, suggesting that the absence of protein modification in the eukaryotic expression system would result in dysfunction of the target protein.

The recombinant proteins FPTD-Grb2-SH2 and FPTD-Grb2-SH2M were expressed in both HER2-negative MDA-MB-231 and HER2-positive MCF-7 cells. Data showed that both these two novel fusion proteins inhibited the growth and migration of the breast cancer cells. The effects of FPTD-Grb2-SH2 and FPTD-Grb2-SH2M on cell migration, were similar while a slight stronger proliferation inhibition by FPTD-Grb2-SH2 was observed. This implies that the reserved sequence FPTD-Grb2-SH2M may still maintain the basic function of the $\mathrm{SH} 2$ domain. Despite that both $\mathrm{SH} 2$ and SH3 domains recognize and specifically bind to their ligands, SH2 domains are major known binding modules for tyrosine-phosphorylated proteins and are essential for breast cancer cell survival (29). Furthermore, compared with FPTD-Grb2-SH2M, FPTD-Grb2-SH2 showed enhanced ability to induce apoptosis in MCF-7 cells. The discrepancy may be explained by the possibility that the pathway structure affected by administration of the proteins resulted in abnormal cell viability. However, we did not estimate the downstream molecules or the pathways of the Grb2-SH2 protein domain involved in the intricate intracellular networks (30), and these aspects need elucidation in further studies. 
It has been reported that the function of Grb2 is closely associated with HER2 and the signaling pathway induced by the HER 2 receptor in human cancer cells $(31,32)$. In the cell apoptosis analysis, unconspicuous sensitivity to FPTD-Grb2-SH2 and FPTD-Grb2-SH2M was shown in the HER2-negative MDA-MB-231 cells, compared with the HER2-positive MCF-7 cells. This indicates that the SH2 domain may function with more specificity to the HER 2 signal pathway during cell apoptosis. This is opposite to previous results (25), which confirmed that the eukaryotic expression system may be helpful for the function of protein in the present study.

In conclusion, the recombinant proteins FPTD-Grb2-SH2 and FPTD-Grb2-SH2M were successfully expressed in the breast cancer cell lines regardless of HER2 phenotype. The SH2 domain may maintain its biological activity alone. Moreover, both the wild-type and the mutant one exhibited the ability to inhibit growth and migration and induce apoptosis of breast cancer cells. This indicated that FPTD-Grb2-SH2 and FPTD-Grb2-SH2M exhibited significant toxicity to breast cancer cells. The proteins expressed in the eukaryocyte may be used for anticancer drug development.

\section{Acknowledgements}

This study was supported by grant no. 30901457 from the National Natural Science Foundation of China.

\section{References}

1. Theriault RL, Carlson RW, Allred C, et al: Breast cancer, version 3.2013: featured updates to the NCCN guidelines. J Natl Compr Canc Netw 11: 753-761, 2013.

2. Zheng S, Bai JQ, Li J, et al: The pathologic characteristics of breast cancer in China and its shift during 1999-2008: a nationalwide multicenter cross-sectional image over 10 years. Int J Cancer 131: 2622-2631, 2012.

3. Tari AM, Hung MC, Li K and Lopez-Berestein G: Growth inhibition of breast cancer cells by Grb2 downregulation is correlated with inactivation of mitogen-activated protein kinase in EGFR, but not in ErbB2, cells. Oncogene 18: 1325-1332, 1999.

4. Roskoski R Jr: The ErbB/HER receptor protein-tyrosine kinases and cancer. Biochem Biophys Res Commun 319: 1-11, 2004.

5. Normanno N, De Luca A, Bianco C, et al: Epidermal growth factor receptor (EGFR) signaling in cancer. Gene 366: 2-16, 2006.

6. Zhang X, Diaz MR and Yee D: Fulvestrant regulates epidermal growth factor (EGF) family ligands to activate EGF receptor (EGFR) signaling in breast cancer cells. Breast Cancer Res Treat 139: 351-360, 2013

7. Saito Y, Furukawa T, Arano Y, Fujibayashi Y and Saga T: Fusion protein based on Grb2-SH2 domain for cancer therapy. Biochem Biophys Res Commun 399: 262-267, 2010.

8. Rexer BN, Ghosh R, Narasanna A, et al: Human breast cancer cells harboring a gatekeeper T798M mutation in HER2 overexpress EGFR ligands and are sensitive to dual inhibition of EGFR and HER2. Clin Cancer Res 19: 5390-5401, 2013.

9. Piechocki MP, Yoo GH, Dibbley SK and Lonardo F: Breast cancer expressing the activated HER2/neu is sensitive to gefitinib in vitro and in vivo and acquires resistance through a novel point mutation in the HER2/neu. Cancer Res 67: 6825-6843, 2007.

10. Bianco R, Melisi D, Ciardiello F and Tortora G: Key cancer cell signal transduction pathways as therapeutic targets. Eur J Cancer 42: 290-294, 2006.
11. Berry DM, Nash P, Liu SK, Pawson T and McGlade CJ: A highaffinity Arg-X-X-Lys SH3 binding motif confers specificity for the interaction between Gads and SLP-76 in T cell signaling. Curr Biol 12: 1336-1341, 2002.

12. Groveman BR, Xue S, Marin V, et al: Roles of the $\mathrm{SH} 2$ and $\mathrm{SH} 3$ domains in the regulation of neuronal Src kinase functions. FEBS J 278: 643-653, 2011.

13. Mayer BJ and Gupta R: Functions of $\mathrm{SH} 2$ and $\mathrm{SH} 3$ domains. Curr Top Microbiol Immunol 228: 1-22, 1998.

14. Daly RJ, Binder MD and Sutherland RL: Overexpression of the Grb2 gene in human breast cancer cell lines. Oncogene 9: 2723-2727, 1994

15. Verbeek BS, Adriaansen-Slot SS, Rijksen G and Vroom TM: Grb2 overexpression in nuclei and cytoplasm of human breast cells: a histochemical and biochemical study of normal and neoplastic mammary tissue specimens. J Pathol 183: 195-203, 1997.

16. Liu Y, Liu Q, Jia W, et al: MicroRNA-200a regulates Grb2 and suppresses differentiation of mouse embryonic stem cells into endoderm and mesoderm. PLoS One 8: e68990, 2013.

17. Di Fulvio M, Henkels KM and Gomez-Cambronero J: Shorthairpin RNA-mediated stable silencing of Grb2 impairs cell growth and DNA synthesis. Biochem Biophys Res Commun 357: 737-742, 2007.

18. Liu BA, Jablonowski K, Shah EE, Engelmann BW, Jones RB and Nash PD: SH2 domains recognize contextual peptide sequence information to determine selectivity. Mol Cell Proteomics 9: 2391-2404, 2010.

19. Songyang Z, Shoelson SE, Chaudhuri M, et al: $\mathrm{SH} 2$ domains recognize specific phosphopeptide sequences. Cell 72: 767-778, 1993.

20. Broadbridge RJ and Sharma RP: The Src homology-2 domains (SH2 domains) of the protein tyrosine kinase p56lck: structure, mechanism and drug design. Curr Drug Targets 1: 365-386, 2000.

21. Machida $\mathrm{K}$ and Mayer BJ: The SH2 domain: versatile signaling module and pharmaceutical target. Biochim Biophys Acta 1747: $1-25,2005$.

22. Pawson T: Protein modules and signalling networks. Nature 373: 573-580, 1995.

23. Mayer BJ, Jackson PK and Baltimore D: The noncatalytic src homology region 2 segment of $a b l$ tyrosine kinase binds to tyrosine-phosphorylated cellular proteins with high affinity. Proc Natl Acad Sci USA 88: 627-631, 1991.

24. Mayer BJ, Jackson PK, Van Etten RA and Baltimore D: Point mutations in the abl SH2 domain coordinately impair phosphotyrosine binding in vitro and transforming activity in vivo. Mol Cell Biol 12: 609-618, 1992.

25. Yin J, Cai Z, Zhang L, et al: A recombined fusion protein PTD-Grb2-SH2 inhibits the proliferation of breast cancer cells in vitro. Int J Oncol 42: 1061-1069, 2013.

26. Watanabe T, Shinohara N, Moriya K, et al: Significance of the Grb2 and son of sevenless (Sos) proteins in human bladder cancer cell lines. IUBMB Life 49: 317-320, 2000.

27. Giubellino A, Burke TR Jr and Bottaro DP: Grb2 signaling in cell motility and cancer. Expert Opin Ther Targets 12: 1021-1033, 2008.

28. Smithgall TE: SH2 and SH3 domains: potential targets for anticancer drug design. J Pharmacol Toxicol Methods 34: 125-132, 1995.

29. Ursini-Siegel J, Hardy WR, Zheng Y, et al: The ShcA SH2 domain engages a 14-3-3/PI3'K signaling complex and promotes breast cancer cell survival. Oncogene 31: 5038-5044, 2012.

30. Dierck K, Machida K, Mayer BJ and Nollau P: Profiling the tyrosine phosphorylation state using SH2 domains. Methods Mol Biol 527: 131-155, 2009.

31. Gril B, Vidal M, Assayag F, Poupon MF, Liu WQ and Garbay C: Grb2-SH3 ligand inhibits the growth of HER2 ${ }^{+}$cancer cells and has antitumor effects in human cancer xenografts alone and in combination with docetaxel. Int J Cancer 121: 407-415, 2007

32. Yu GZ, Chen Y and Wang JJ: Overexpression of Grb2/HER2 signaling in Chinese gastric cancer: their relationship with clinicopathological parameters and prognostic significance. J Cancer Res Clin Oncol 135: 1331-1339, 2009. 